FORMATION Formation emploi

Revue française de sciences sociales

150 | avril-juin 2020

Former aux "petits" métiers : regards internationaux

\title{
Les Baléares : quand le soutien relationnel favorise la poursuite des études en formation professionnelle de base
}

The Balearic Islands: When relational support promotes the pursuit of studies in basic vocational training

Eine beziehungsorientierte Unterstützung, welche die Fortsetzung der beruflichen Grundausbildung auf den Balearen begünstigt

Las Baleares : cuando el apoyo relacional promueve la continuación de los estudios de Formación Profesional Básica

Caterina Thomàs-Vanrell, Antoni Cerdà-Navarro et Francesca Salvà-Mut

\section{OpenEdition}

Journals

Édition électronique

URL : https://journals.openedition.org/formationemploi/8218

DOI : 10.4000/formationemploi.8218

ISSN : 2107-0946

Éditeur

La Documentation française

Édition imprimée

Date de publication : 8 juillet 2020

Pagination : 123-144

ISSN : 0759-6340

Référence électronique

Caterina Thomàs-Vanrell, Antoni Cerdà-Navarro et Francesca Salvà-Mut, « Les Baléares : quand le soutien relationnel favorise la poursuite des études en formation professionnelle de base », Formation emploi [En ligne], 150 | avril-juin 2020, mis en ligne le 02 janvier 2022, consulté le 06 janvier 2022. URL : http://journals.openedition.org/formationemploi/8218 ; DOI : https://doi.org/10.4000/ formationemploi.8218 


\title{
Les Baléares : quand le soutien relationnel favorise la poursuite des études en formation professionnelle de base
}

\begin{abstract}
ThOMÀs-VANRELl CATERINA
Enseignante-chercheure en sociologie. Membre du groupe de recherche Inégalités, Genre et Politiques publiques. Collaboratrice du groupe de recherche Éducation et Citoyenneté, de

I'Université des Îles Baléares

Cerdà-Navarro Antoni

Chercheur collaborateur en sciences de l'éducation du groupe de recherche Éducation et Citoyenneté, de l'Université des lles Baléares

Salvà-Mut Francesca

Enseignante-chercheure permanente en sciences de l'éducation à la Faculté d'Éducation de l'Université des îles Baléares. Elle y est membre de l'équipe de recherche Éducation et

Citoyenneté
\end{abstract}

Résumé

Les Baléares : quand le soutien relationnel favorise la poursuite des études en formation professionnelle de base

Larticle analyse l'intention d'abandonner les études de formation professionnelle des filières de bas niveau d'exigence aux Îles Baléares. Il compare la cohorte d'élèves qui ont exprimé leur intention d'abandonner leur formation quelques mois après le début des cours et ceux qui ne l'ont pas envisagé. Cette analyse est menée à l'aune de leurs caractéristiques sociodémographiques et à la lumière du concept "d'engagement scolaire ". Les résultats révèlent une corrélation de ce dernier avec les contextes de vie, les parcours scolaires des jeunes et leurs attentes et motivations vis-à-vis de leur formation.

Mots clés : enseignement technique-professionnel, abandon des études, jeune en difficulté, accompagnement scolaire, réseau social, Espagne

Abstract

The Balearic Islands: When relational support promotes the pursuit of studies in basic vocational training

The article analyses the intention to abandon low-level vocational training studies in the Balearic Islands. The study concerns students who manifested their intention to drop out a few months after the start of the school year and those who did not consider abandonment. We compare socio-demographic characteristics and educational paths in 
relation to the concept of "school engagement". The results show a relationship amongst life contexts, educational paths and the expectations and motivations of young people to pursue their studies.

Keywords: technical \& vocational education, drop out, young person in difficulty, school support, social network, Spain

Journal of Economic Literature: I 21

Traduction : auteur.e.s.

\section{Introduction}

Afin de mieux appréhender la situation des jeunes, notamment en termes de besoins en compétences et de formation susceptibles d'améliorer leurs perspectives d'insertion professionnelle futures, nous nous interrogeons ici sur les facteurs relationnels et le soutien social influençant la poursuite d'études.

Cet article présente les résultats de la première vague d'enquête d'une étude longitudinale ${ }^{1}$ portant sur les facteurs conditionnant la poursuite d'études en formation professionnelle de jeunes originaires de Majorque (Mallorca-Espagne), dans les filières de bas niveau d'exigence ${ }^{2}$.

Ces jeunes en formation professionnelle de base (FPB) (cf. encadré 1) se trouvent dans un contexte social particulier, caractérisé par un environnement socio-économique principalement axé sur le tourisme. Jusqu'au début de la récession économique de 2008, les jeunes accédaient aisément à un emploi non qualifié, ce qui a favorisé un taux de scolarisation inférieur à la moyenne de l'ensemble de l'État espagnol (MECD ${ }^{3}$, 2010). Mais ce taux s'est accru à partir de 2009, en raison de la difficulté croissante à trouver un emploi. Le faible niveau de qualification d'une partie de la population jeune persiste cependant depuis deux décennies. Dès lors, l'étude des facteurs influençant l'intention d'abandonner la formation devient prioritaire afin d'améliorer une perspective d'insertion professionnelle digne, notamment des populations en situation de vulnérabilité.

1. L’enquête a été réalisée en trois vagues successives sur les années scolaires 2015/16, 2016/17 et 2017/18. Dans cet article, nous présentons les résultats de la première année d'enquête, qui feront objet d'un travail successif visant à montrer la dimension longitudinale de l'étude.

2. Filières de formation professionnelle destinées aux jeunes n'ayant pas obtenu leur diplôme de l'enseignement secondaire obligatoire. En Espagne, on utilise le terme de Formation professionnelle de base (FPB).

3. Ministerio de Educación y Ciencia (ministère de l'Éducation et et de la Science). 


\section{Encadré 1 : Formation Professionnelle de Base (FPB) en Espagne}

La formation professionnelle de base constitue « le » parcours que le système éducatif espagnol propose aux élèves qui n'obtiennent pas ou dont on présume qu'ils n'obtiendront pas le diplôme de l'Éducation secondaire obligatoire (ESO). Cette formation est destinée à un public dont le parcours est marqué par l'échec scolaire. Elle n'est pas véritablement choisie par les élèves, ces derniers étant dirigés vers ces formations par le système d'orientation des établissements de l'ESO.

La FPB a été introduite pour la première fois l'année scolaire 2014-15, suite à la Loi Organique de 2013 pour l'Amélioration de la Qualité de l'Éducation (LOMCE).

La FPB trouve son origine dans les programmes de garantie sociale (PGS) instaurés par la Loi sur l'Organisation Générale du Système Éducatif (LOGSE) (1990). Cette loi a allongé la scolarité obligatoire de 14 à 16 ans, établi un tronc commun complet entre les 12-16 ans et un diplôme unique à la fin de l'ESO (le Diplôme de l'Enseignement Secondaire ObligatoireGESO). Ce diplôme est devenu obligatoire pour l'accès à l'enseignement secondaire postobligatoire dans la filière générale (Baccalauréat) et dans la filière professionnelle (Formation Professionnelle-FPGM, équivalent au BEP - brevet d'études professionnelles - en France, et du CFC - Certificat fédéral de capacité - en Suisse).

Dans ce contexte, le PGS est un dispositif de seconde chance. II vise à faciliter l'insertion dans la vie active et / ou la réintégration dans le système éducatif réglementé des jeunes de 16 à 21 ans non scolarisés n'ayant pas obtenu le diplôme de l'ESO. Les PGS ont fait l'objet de diverses modifications et changements de nom au cours des lois successives sur l'éducation. Ils ont ainsi été renommés Programmes d'Initiation Professionnelle (PIP) par la Loi Organique sur la Qualité de l'Éducation - LOCE (2002), et Programmes de Qualification Professionnelle Initiale (PCPI) par la Loi Organique sur l'Éducation-LOE (2006).

La FPB présente des caractéristiques spécifiques qui, avec les situations décrites ci-dessus, peuvent favoriser certaines des conséquences suivantes pour les jeunes (Marhuenda-Fluixà $\mathrm{F}$. \& al., 2015) :

- L'âge d'entrée est abaissé à 15 ans pour tous les élèves (et non plus à titre exceptionnel).

- Ceux qui l'intègreront n'obtiendront pas le certificat d'ESO, mais une qualification de niveau 1 de l'EFP.

- Cette mesure peut être considérée comme discriminante ; en effet, ce diplôme est obtenu à l'âge de la scolarité obligatoire et ignore le principe de l'inclusivité (collège unique) ${ }^{(*)}$ qui fait partie intégrante du système scolaire depuis 1990.

- Cela introduit également une rupture dans le consensus qui veut que l'accès à la voie académique ou à la voie professionnelle au niveau post-obligatoire soit conditionné à l'obtention du diplôme de l'enseignement obligatoire.

$\left.{ }^{*}\right)$ : Le terme « inclusivité » (renvoyant au collège unique en France) est la traduction proposée de " comprehensivity » et fait référence à la « branche commune », sans différencier les itinéraires tout au long de l'enseignement obligatoire. En espagnol, il est généralement traduit par "escuela comprensiva », qui est une traduction littérale de l'anglais. 
L'article comporte cinq parties. L'introduction pose le cadre de l'étude, les tendances statistiques qui justifient le choix de la population étudiée et la problématique de recherche. La deuxième partie présente le contexte et le cadre théorique. La troisième a trait à la méthodologie. Enfin, les résultats sur les facteurs influençant les intentions d'abandonner les études seront présentés et discutés dans la quatrième partie. Ils y seront détaillés en deux sous-parties : la première présente les intentions d'abandon des études pour expliquer l'engagement scolaire et l'autre montre, de manière originale, la faible influence des caractéristiques sociodémographiques sur l'engagement scolaire des jeunes. Ici, l'engagement scolaire est le processus qui permet aux élèves de créer un attachement individuel au système scolaire et à toutes les valeurs, pratiques, activités et relations sociales que cela implique. Nous reviendrons sur la définition et l'état de la littérature autour de ce concept dans la deuxième partie de l'article.

La contribution révèle ainsi que les facteurs relationnels expliquent partiellement les intentions d'abandonner les études. On note que les relations élèves-enseignants influent sur les possibilités de renforcer la persévérance scolaire. Et au final, l'appartenance sociale conditionne le soutien familial à l'égard des jeunes et les attentes de leurs parents en termes de réussite.

Aborder les dynamiques de décrochage de la formation professionnelle nécessite une clarification conceptuelle. Dans la terminologie francophone, on évoque souvent le décrochage ${ }^{4}$ lorsqu'un élève quitte l'institution scolaire, abandonne ses études, arrête le cursus avant qu'il ne soit achevé (ministère de l'Éducation nationale et de la Jeunesse, 2020)

"... selon le code de l'éducation, être décrocheur, c'est ne pas avoir terminé avec succès le cycle de formation de second cycle du second degré dans lequel le jeune sétait engagé. Au niveau européen, la notion de décrochage correspond à la proportion de personnes de 18 à 24 ans qui ont seulement le niveau de l'enseignement secondaire inférieur ou un niveau moins élevé et sont sorties du monde de l'éducation ou de la formation. Le cadre stratégique 'Éducation et formation 2020' a retenu pour objectif de faire descendre cette proportion en-dessous de $10 \% »$.

Dans le cadre de la "Stratégie Éducation et Formation 2020 ", la lutte contre le décrochage est un des objectifs prioritaires des politiques publiques de l'Union européenne et d'une grande partie de ses États-membres. Au sein des politiques publiques d'éducation, cette lutte contre le décrochage est motivée par deux objectifs : d'une part, éviter les effets individuels indésirables sur les opportunités futures de l'élève ; d'autre part, réduire les conséquences de la généralisation des bas niveaux d'éducation chez les jeunes, susceptible

4. Tout au long de l'article, nous utiliserons alternativement le terme décrochage ou abandon comme synonymes.

5. Voir les définitions des termes et indicateurs statistiques utilisés sur le site du ministère de l'Education nationale : https://www.education.gouv.fr/les-definitions-des-termes-et-indicateurs-statistiques-de-l-education-nationale-5123 
d'engendrer des problèmes économiques et sociaux globaux. Le premier objectif, individuel, prend en compte les risques élevés de chômage, de pauvreté, d'exclusion sociale et leurs effets sur la santé. Le deuxième objectif s'intéresse aux conséquences sociétales se concrétisant par un taux de chômage des jeunes élevé, un coût social plus important, une pénurie de compétences de la force de travail et une compétitivité moindre dans l'économie mondialisée contemporaine (Araujo \& al., 2014).

Selon les données les plus récentes (2018), le taux moyen de décrochage, en Europe, est de 10,6\%, contre 17,9\% en Espagne et 24,4\% dans les îles Baléares (MEFP', 2019). Ces chiffres témoignent d'une grande disparité entre les pays européens. L'Espagne est le pays de l'UE-28 qui enregistre le plus fort taux de décrochage des 18-24 ans, et aux Baléares, ce taux est le plus élevé de tout le pays puisque, en 2018, le taux de décrochage des hommes est de $27 \%$, contre $21,7 \%$ pour les femmes.

Parmi les facteurs de risque d'abandon d'une formation professionnelle à bas niveau d'exigence, évoqués dans d'autres études, on retrouve la vulnérabilité sociale, le fait d'être un homme, l'appartenance ethnique (Mackay, 2005), ou encore le redoublement (Jimerson \& al., 2002 ; Stearns \& al., 2007). Le redoublement, pratique fréquente au sein du système éducatif espagnol, est considéré comme préjudiciable à l'obtention d'un diplôme (Cordero \& al., 2014 ; Salina \& Santín, 2012).

La plupart des facteurs de risque évoqués dans la littérature sur le décrochage scolaire peuvent s'appliquer aux Baléares. Cependant, les caractéristiques du système éducatif, tels que l'accompagnement des jeunes dans leur parcours et la forte fréquence du redoublement, engendrent la surreprésentation de jeunes possédant certaines caractéristiques sociodémographiques autres que le statut social et la situation économique de la famille (Christenson \& Thurlow, 2004 ; Cerdà-Navarro \& al., 2017 ; Salvà-Mut \& al., 2019 ; Nouwen \& Clycq, 2019). Ainsi, les analyses réalisées dans des travaux précédents (CerdàNavarro, 2019 ; Cerdà-Navarro \& al., 2020) révèlent un biais de sélection au sein de cette population : incidence des redoublements, forte présence de populations issues de minorités ethniques, jeunes aux trajectoires familiales marquées par une situation financière instable, ou encore présence majoritaire de garçons dans ces parcours.

Étant donné l'impact de certaines inégalités sociales sur les résultats scolaires, la recherche en éducation a souvent cherché à expliquer les origines de la variance au sein des groupes à risque de décrochage (Christenson \& Thurlow, 2004). Pour expliquer la dynamique des résultats académiques, la littérature sur le "Student engagement " distingue trois dimensions différentes et articulées du concept : respectivement, les dimensions cognitive, émotionnelle et comportementale (Appleton \& al., 2008 ; Fredrick \& al., 2004 ; Reschly \& Christenson, 2012).

6. Ministerio de Educación y Formación Profesional (ministère de l'Education et de la Formation professionnelle). 
Inspirée par le contexte des politiques éducatives présenté plus haut, et à l'aune de la recherche visant à modifier la focale parmi les facteurs de risque évoqués dans d'autres recherches (Cerdà-Navarro \& al., 2017 ; Salvà-Mut \& al., 2019), l'étude répond à la problématique suivante : les soutiens relationnels dont les jeunes bénéficient peuventils favoriser l'engagement scolaire dans les centres de formation professionnelle sur l'ensemble de l'île ${ }^{7}$ où se concentrent les populations d'élèves ayant un risque élevé de décrochage?

Dans les parties un et deux, nous présentons les variables et les modalités d'analyse nous permettant d'analyser l'impact du réseau relationnel (amis, famille et enseignants) sur le soutien social et donc sur l'engagement scolaire des jeunes.

\section{Contextualisation et cadre théorique}

Bien que la force explicative du concept d'engagement scolaire soit largement reconnue dans les travaux sur le décrochage, les dimensions spécifiques (comportementales, cognitives et relationnelles) ${ }^{8}$ et leur articulation (Appleton \& al., 2008) suscitent encore un large débat. Il reste encore beaucoup à apprendre sur le lien entre l'engagement scolaire des élèves, les caractéristiques sociodémographiques et professionnelles et le soutien des parents, des amis ou des enseignants (Wang \& Eccles, 2012 ; Elffers \& al., 2012).

La littérature existante (Appleton \& al., 2008) permet de mieux appréhender la variation de l'engagement scolaire parmi les jeunes à risque de décrochage scolaire et de démêler les relations spécifiques entre le statut à risque des jeunes, le soutien social et les comportements en termes d'engagement éducatif. Nous présentons maintenant les concepts d'engagement scolaire et de soutien social.

7. Il s'agit des centres de formation professionnelle d'accueil pour des jeunes des filières de base. Les distances courtes sur le territoire insulaire de Mallorca font que les centres de formation sont tous assimilables à des centres urbains, avec une forte densité de population accueillie.

8. Certains auteurs (Appleton \& al., 2008 ; Nouwen \& Clycq, op. cit.) incluent aussi la dimension académique comme critère d'évaluation de l'engagement scolaire. Nous considérons les trois dimensions citées pour décrire la notion d'engagement dans l'élaboration du questionnaire. Dans l'article, les analyses incluent uniquement la dimension relationnelle (le terme d'affectivité est aussi utilisé dans la littérature sur l'engagement). Nous avons choisi de réaliser l'analyse sur l'engagement relationnel en raison de sa pertinence par rapport à la poursuite des études. Plusieurs travaux établissent que l'abandon est un long processus durant lequel le détachement affectif et cognitif qui se construit dans les phases initiales, débouche finalement sur un détachement comportemental (mauvaise conduite, absentéisme, indiscipline, etc.). Par conséquent, le lien émotionnel serait un moyen de gérer les premières étapes du processus d'abandon (Archambault \& al., 2009). 


\subsection{L'engagement scolaire comme concept multidimensionnel et dynamique}

L'engagement scolaire est un concept assez nouveau (Appleton \& al., 2008). Au cours des années 2010, ce concept est apparu comme l'un des facteurs explicatifs les plus importants du décrochage scolaire au niveau individuel (Barry \& Reschly, 2012 ; Reschly \& Christenson, op. cit.). L'engagement scolaire se caractérise par son caractère dynamique et sa nature multidimensionnelle.

Il permet d'analyser un processus graduel pouvant éventuellement conduire à un retrait émotionnel et comportemental de l'école. Abandonner la formation avant d'obtenir un diplôme résulte d'un processus dans la durée, lié à un désengagement définitif ou à des interruptions successives qui se soldent par un abandon (Reschly \& Christenson, Ibid.). La notion de "socialisation professionnelle " permet également d'éclairer l'engagement scolaire et son interprétation dans les parcours de formation et les itinéraires d'insertion professionnelle des jeunes. La socialisation professionnelle (Cohen-Scali, 2003) comporte deux dimensions : la socialisation du travail, qui correspond à l'expérience sociale vécue dans la famille et dans l'éducation, dès l'enfance ; la socialisation par le travail, relative à l'expérience professionnelle des jeunes adultes.

\subsection{Le soutien social comme indicateur de décrochage scolaire}

Plusieurs approches de recherche en sciences sociales ont identifié et étudié les facteurs associés à l'engagement scolaire et au décrochage, incluant la famille, les amis et le soutien des enseignants ${ }^{9}$ (Brewster \& Bowen, 2004 ; Elffers \& al., 2012 ; Roorda $\&$ al., 2011). Rosenfeld \& al. (2000) ont comparé les effets du soutien scolaire des parents, pairs et formateurs sur différents résultats scolaires. Les élèves bénéficiant d'un fort soutien social de la part de ces trois entités consacrent plus de temps aux études, évitent les comportements anti-sociaux, poursuivent davantage leurs études, et font preuve de plus d'autonomie et d'engagement.

Le soutien social est devenu un facteur largement étudié dans les recherches sur les événements biographiques des individus et au sein de nombreuses disciplines des sciences sociales (Passy, 2002 ; Ferrand, 2006 ; Knox, Savage \& Harvey, 2006 ; Martí \& Lozares, 2008).

Le soutien social peut s'expliquer comme la capacité des individus à trouver, dans leurs interactions sociales formelles et informelles, de l'aide ou des ressources émotionnelles, instrumentales, informationnelles ou matérielles (Ferrand, op. cit.). Cette perspective de recherche fait référence à la capacité des individus à activer des sources

9. Certains auteurs (Fall \& Roberts, 2012) suggèrent que lorsque les enseignants s'intéressent aux élèves, louent leurs efforts et encouragent l'émergence de bonnes relations dans le centre éducatif, cela influence directement l'estime de soi des étudiants et leur engagement (Beaton \& Beaton, 2019). 
diverses dans leur entourage. Dans cette contribution sur l'engagement dans la formation, nous étudierons l'impact favorable des soutiens relationnels dont bénéficient les jeunes sur l'issue des transitions sociales et professionnelles.

\section{La méthodologie utilisée}

Après avoir présenté l'échantillon et le terrain d'enquête, nous préciserons les critères d'analyse, puis les variables d'étude.

\section{1. Échantillon et terrain d'enquête}

Parmi l'échantillon ( $\mathrm{N}=354), 31,6 \%$ sont des femmes et $22 \%$ sont des étrangers ; l'âge moyen est de 15,9 ans (écart-type $=0,70$ ). La plupart des jeunes ont entre 15 et 16 ans : $25 \%$ ont 15 ans, $60 \%$ ont 16 ans, $14 \%$ ont 17 ans et $1 \%$ ont 18 ans.

Un quart de la population enquêtée $(24,9 \%)$ a une mère migrante. Le niveau d'études des mères est majoritairement faible : plus des deux tiers des jeunes $(69,6 \%)$ ont une mère ayant un niveau de formation de base ou inférieur au baccalauréat ; $21 \%$ ont des mères qui possèdent un niveau d'études moyen (baccalauréat ou formation professionnelle moyenne) ; enfin, 9,4 \% ont une mère qui a suivi des études supérieures (formation professionnelle supérieure ou études universitaires).

La population étudiée éprouve des difficultés économiques moyennes dans la vie quotidienne : la moitié des jeunes et de leurs familles ne rencontrent pas de difficulté économique ; presque un quart $(23,3 \%)$ rencontrent quelques difficultés et plus d'un quart $(27,7 \%)$ éprouvent de grandes difficultés à subvenir à leurs besoins économiques.

La majorité des jeunes enquêtés $(87,9 \%)$ ont redoublé au moins une année scolaire au cours de leur formation secondaire obligatoire. Parmi les facteurs de risque, on note qu'un cinquième des jeunes $(20,9 \%)$ ont eu envie d'abandonner deux ou trois mois après le début de leur formation.

Bien qu'il ait été démontré que l'origine sociale ou le lieu de naissance peuvent contribuer à l'abandon (Rumberger, 2008), plusieurs études s'interrogent sur la possibilité d'appréhender l'engagement scolaire des jeunes au-delà de leur origine sociale et de leur responsabilité individuelle. Une bonne façon de considérer les itinéraires et les perceptions des élèves en tenant compte de la diversité des situations est de prendre en compte la manière dont l'engagement scolaire est conditionné aux relations établies avec les amis, les enseignants et la famille.

La possibilité d'accéder aux ressources permettant de suivre des études dépendra, en partie, du contexte d'origine. Cependant, pour les élèves d'origine sociale populaire qui 
ont moins accès à des formes de capital social plus larges, complexes et diverses, l'engagement à l'école dépendra notamment du soutien des professionnels de l'enseignement (Fredrick \& al., 2004 ; Furrer \& Skinner, 2003). Reschly \& Christenson (2012) soulignent la difficulté de réduire le décrochage et de favoriser la réussite scolaire pour les jeunes aux caractéristiques d'appartenance sociale à risques (sexe, lieu de naissance ou milieu social). Dès lors, ces auteurs proposent de se centrer sur l'intervention sociale. Il s'agit d'agir sur l'engagement scolaire et ses dimensions (relationnelle, comportementale et cognitive) permettant d'influencer la réalité de la vie des jeunes et leur activité dans le cadre de la formation.

Les données ont été recueillies à partir d'un questionnaire papier auto-administré dans les salles de classe. Les élèves ont rempli le questionnaire deux ou trois mois après le début de l'année scolaire ${ }^{10}$.

Il s'agit d'une enquête sur un échantillon de 354 élèves de première année (14-18 ans), statistiquement représentatif des 990 élèves de la formation professionnelle de base (FPB) à Mallorca (Espagne), enquêtés pendant leur première année scolaire 2015-2016 ${ }^{11}$.

L'échantillon est constitué de 34 classes de première année de FPB, réparties dans les dixneuf centres de formation professionnelle de Majorque ayant participé à l'étude.

Nous avons vérifié la proportionnalité de l'échantillon par rapport au poids de chaque groupe professionnel proposé dans les centres de formation (Voir Tableau 1 en Annexe de la version électronique). Cependant, certains groupes professionnels sont légèrement sous-représentés dans l'échantillon (par exemple, les formations en administration et gestion ou en hôtellerie et tourisme).

Le questionnaire a été conçu ad hoc pour cette étude, qui repose principalement sur l'articulation entre le contexte social (la famille, les parents, le milieu de formation et la communauté), l'engagement (cognitif, comportemental et émotionnel), les résultats académiques des élèves (Reschly \& Christenson, op. cit.), et enfin l'examen de différents instruments pour la mesure et l'opérationnalisation de l'engagement ainsi que les différentes dimensions sociales étudiées. Le questionnaire qui en a résulté s'est fortement inspiré d'autres travaux concernant la notion d'engagement ${ }^{12}$.

10. L'étude a été approuvée par le comité d'éthique de la recherche de l'Université.

11. Cela représente une marge d'erreur dans l'échantillonnage de $+-4,2 \%$, avec un intervalle de confiance de $95 \%$ dans la condition la plus défavorable $(p=q=0,5)$. L'échantillon initialement prévu était légèrement plus important ; cependant, au cours de l'étude de terrain, les chercheurs ont constaté un fort taux d'absentéisme chez les élèves devant y participer.

12. Concrètement, il s'agit des contributions du Student Engagement Instrument (SEI), utilisé dans le projet "Check \& Connect " (Appleton, 2012), basées sur l'évaluation du système d'aide aux décrocheurs potentiels (Trousse d'évaluation des Décrocheurs Potentiels-TEDP), employé au Québec (Janosz \& al., 2007) et d'une étude sur l'absentéisme dans l'enseignement secondaire en France (Lannegrand \& al., 2012). 


\subsection{Critères d'analyse}

Les résultats sont issus d'une analyse quantitative centrée sur le soutien relationnel que les jeunes élèves reçoivent de leurs parents, amis et enseignants, et qui peut influencer l'intention de poursuivre les études de FPB.

Le travail se structure en deux objectifs spécifiques, consistant à :

1) Analyser la dimension relationnelle affective de l'engagement, à l'aune de quatre facteurs : a) les relations avec les enseignants ; b) les relations avec les amis ; c) la disponibilité de la famille; d) et les attentes de la famille et leur lien avec les variables sociodémographiques de cette population (le sexe des jeunes, le lieu de naissance, les difficultés économiques familiales, le lieu de naissance de la mère et le niveau d'éducation des parents);

2) Comprendre l'intention de poursuivre des études de FPB d'un groupe d'élèves en fonction du soutien relationnel reçu des parents, des amis dans le cadre des études et des loisirs, et des enseignants.

\subsection{Les variables d'études}

Les variables explicatives considérées pour réaliser cette analyse, qui visent à qualifier les aspects affectifs de l'engagement ${ }^{13}$, sont structurées autour de quatre facteurs relationnels regroupant vingt-trois items.

Pour valider l'influence des facteurs relationnels dans les parcours de formation des jeunes, nous considérons plusieurs variables indépendantes : une variable centrée sur la mesure des conditions de poursuite ou d'abandon des études; et un ensemble de caractéristiques sociodémographiques (le sexe des jeunes, le lieu de naissance, les difficultés économiques familiales, le lieu de naissance de la mère et le niveau d'éducation des parents).

\section{- La dimension affective}

Le premier bloc de variables fait référence à la dimension affective de l'engagement, qui se concrétise par quatre facteurs relationnels : la relation avec les enseignants, la relation avec les amis, le soutien, la disponibilité de la famille, et les attentes de la famille ${ }^{14}$.

Pour le premier facteur, la relation avec les enseignants, les sept items analysés $(\boldsymbol{a}=0,820)$ concernent les relations avec les enseignants du centre (exemple : "J'aime parler aux professeurs de mon collège ou lycée »). Une valeur élevée témoigne d'une bonne relation entre les élèves et les enseignants.

13. Nous avons utilisé un regroupement des items selon une sélection déjà validée dans d'autres recherches sur la poursuite des études de formation professionnelle de base (Salvà-Mut \& al., 2019 ; Cerdà-Navarro \& al., 2019 ; Cerdà-Navarro, 2019).

14. Le choix du nombre d'items pour chaque facteur dépend de la valeur d'alfa de crombach des items, calculés sur les données de l'enquête. 
Le second facteur, quant à lui, prend en compte les relations avec les amis ou camarades de classe ; les analyses se centrent sur cinq items $(\alpha=0,713)$ (exemple : "J'ai des amis au collège ou lycée »). Les valeurs élevées révèlent une bonne relation avec les amis.

Concernant les interactions liées aux relations parentales, les analyses s'appuient sur les six items concernant la disponibilité et le soutien familial $(a=0,830)$ (exemple : "Je peux compter sur mes parents si j’ai des problèmes au collège ou lycée ").

Enfin, on compte quatre items pour les attentes académiques des parents $(\alpha=0,670)$ envers leurs enfants (exemple : "Mes parents souhaitent que je continue le plus longtemps possible mes études »). Les valeurs élevées indiquent un souhait de formation important de la part des parents.

- Abandonner ou poursuivre les études

Le second bloc de variables concerne l'abandon ou la poursuite des études. Il répond à la question suivante (avec une réponse à double option : oui ou non) : "Avez-vous pensé à abandonner les études que vous suivez actuellement?".

La procédure statistique d'analyse a consisté à réaliser une comparaison des moyennes d'échantillons indépendants (test- $t)^{15}$ pour analyser les différences entre les groupes (variables catégorielles) par rapport aux divers facteurs liés à l'engagement des élèves (variables métriques).

\section{La relation élève-enseignant renforce 3 l'engagement scolaire}

Les facteurs influençant la poursuite des études sont multiples. Ils sont partiellement liés à la possibilité de trouver du soutien auprès des amis ou des parents, et majoritairement de la part des enseignants. Les résultats se structurent en deux parties : une première partie relative à l'engagement versus l'intention d'abandonner les études, et une seconde qui synthétise l'influence des caractéristiques sociodémographiques sur l'engagement scolaire des jeunes.

15. Le test statistique utilisé "T-test " ou " $\mathrm{t}$-test » (Test de Student) pour des échantillons indépendants s'appelle ainsi parce que différents échantillons sont comparés (par exemple, pour la variable sexe, les hommes et les femmes forment deux échantillons ; en ce qui concerne l'origine, il y a les natifs et les immigrés. Les moyennes sont les mesures permettant de comparer les variables qui se réferent à l'engagement et les groupes qui sont comparés constituent les variables catégorielles (sexe : hommes et femmes; origine : natifs et immigrés, etc.). 


\subsection{Engagement versus intention d'abandonner les études}

L'analyse des facteurs liés au concept d'engagement relationnel (relations familiales, avec les enseignants et avec les amis) a été réalisée, dans un premier temps, à l'aune des opinions sur les intentions de décrochage.

Elle a consisté à enregistrer les opinions des élèves, amenés à répondre sur le degré de soutien reçu de la part des amis, de la famille et des enseignants. Les réponses étaient codées de 0 à 3, 0 représentant le moins de soutien et 3 le plus. Les chiffres mentionnés dans les tableaux font référence à la moyenne pour chaque question ou groupement de questions (Voir Tableau 2 en Annexe de la version électronique).

Le score des relations avec les enseignants est en moyenne proche de 2 , sachant que le score relatif aux attentes des parents sur la réussite est le plus élevé $(2,6)$, suivi de celui du soutien de la famille $(2,2)$ et du score de la relation avec les amis $(2,1)$.

Les opinions sur l'importance qu'exerce l'aspect relationnel sur l'engagement présentent toujours la même hiérarchie pour toutes les caractéristiques sociodémographiques (voir Tableaux 1 à 4 dans l'article). Ainsi, les opinions sur la famille enregistrent les valeurs les plus élevées, suivies des opinions relatives aux amis et enfin aux enseignants.

Notons que s'agissant des relations amicales, les items les moins bien notés par les jeunes sont liés au "partages d'opinions avec leurs pairs ", et que le plus noté est corrélé avec le fait "d'avoir des amis au lycée".

Nous observons une interaction difficile entre les jeunes et leurs enseignants. En effet, "parler aux enseignants" est l'item le moins bien noté. Cependant, les jeunes considèrent qu'ils sont soutenus par leurs professeurs (l'item qui obtient le score le plus élevé est le fait de considérer que "les professeurs sont disponibles sils en ont besoin "). Les jeunes s'accordent sur le fait que "leur famille est disponible quand ils en ont besoin " (item le plus noté) ; en revanche, le fait d'informer les parents sur les dossiers à rendre ou les examens est un facteur de moindre importance.

Les opinions concernant la relation avec les enseignants sont le seul facteur qui présente des écarts significatifs selon que les jeunes ont envisagé, ou pas, au moins une fois, d'abandonner leurs études.

Des différences statistiques s'observent entre les élèves qui ont songé à abandonner leurs études $(22 \%)$ et les autres $(78 \%)^{16}$, en termes de relation avec les enseignants (voir Tableau 1 dans l'article). Ainsi, les élèves qui envisagent d'abandonner leur formation entretiennent une piètre relation avec les enseignants et ont en général une moins bonne perception de ces derniers (considérés comme moins disponibles, moins à l'écoute ; les jeunes déclarent ne pas apprécier de leur parler).

16. Pourcentage valide, onze élèves n’ayant pas donné de réponse à cette question. 
Les tests de comparaison des moyennes révèlent une combinaison de cinq caractéristiques des relations avec les enseignants pour les jeunes non désireux d'abandonner leurs études. Il s'agit, par ordre d'importance, du fait que les enseignants soient disponibles pour eux quand ils en ont besoin, que la relation avec les professeurs soit ouverte et honnête, le fait de se sentir bien traité par les enseignants, leur disponibilité, et le fait que les élèves apprécient d'échanger avec leurs enseignants (cela renvoie à l'item "J'aime parler aux professeurs ").

Les autres dimensions analysées (relations avec les pairs, disponibilité de la famille et attentes de la famille en termes de scolarité) ne reflètent pas de différences statistiquement significatives entre les élèves qui pensaient abandonner et ceux qui ne l'envisageaient pas. Nos travaux en cours sur ces questions confortent l'idée selon laquelle certaines caractéristiques sociodémographiques et du parcours scolaire des jeunes renforcent les intentions de décrochage pour les filières de FP moyennes ; dans le cas des filières de FPB présentées dans cet article, cette influence est même majorée (Cerdà-Navarro, 2017 ; Cerdà-Navarro \&al., 2019).

Tableau 1. Scores selon les critères conditionnant l'intention d'abandonner les études. Comparaison de moyennes entre ceux qui envisagent ou pas d'abandonner

\begin{tabular}{|c|c|c|c|}
\hline & \multicolumn{3}{|c|}{$\begin{array}{l}\text { Les jeunes ont envisagé au moins une fois } \\
\text { d'abandonner en cours d'études }\end{array}$} \\
\hline & Oui & Non & Total \\
\hline Mes professeurs sont disponibles quand j'en ai besoin & 2,00 & 2,33 & 2,26 \\
\hline Le personnel enseignant de mon collège ou lycée écoute les élèves & 1,77 & $2,15^{*}$ & 2,06 \\
\hline $\begin{array}{l}\text { Le personnel enseignant de mon établissement s'intéresse à moi non } \\
\text { seulement en tant qu'élève, mais aussi en tant que personne }\end{array}$ & 1,70 & 1,90 & 1,86 \\
\hline En général, mes professeurs sont ouverts et honnêtes avec moi & 1,82 & $2,18^{*}$ & 2,11 \\
\hline $\begin{array}{l}\text { En général, le personnel enseignant de mon collège ou lycée traite les } \\
\text { élèves d'une manière adéquate }\end{array}$ & 1,86 & 2,16 & 2,09 \\
\hline J'aime parler aux professeurs de mon collège ou lycée & 1,42 & $1,89^{*}$ & 1,79 \\
\hline $\begin{array}{l}\text { Dans mon collège ou lycée, la plupart des enseignants se préoccupent } \\
\text { de leurs élèves }\end{array}$ & 1,89 & 2,04 & 2,00 \\
\hline Relations avec les enseignants (total) & 1,80 & $2,09 *$ & 2,03 \\
\hline Total & $\begin{array}{c}22 \% \\
(n=74\end{array}$ & $\begin{array}{c}78 \% \\
(n=269)\end{array}$ & $\begin{array}{c}100 \% \\
(n=343)\end{array}$ \\
\hline
\end{tabular}

Note : * les valeurs de la même ligne comportant un * et grisées sont significativement différentes à $p<, 05$ dans le test d'égalité bilatérale pour les moyennes en colonne. Les cellules sans indice ne sont pas incluses dans le test. Les tests supposent des variances égales.

Source : auteur.e.s. 


\subsection{Engagement et caractéristiques sociodémographiques}

Nous abordons ici l'influence des caractéristiques sociodémographiques sur l'engagement affectif des jeunes. Le seul élément notable concerne l'aspect des relations avec les pairs : les garçons ont plus d'amis que les filles dans leur centre de formation (score de 2,63 pour les garçons, contre 2,49 pour les filles). La littérature relative à l'analyse des réseaux personnels confirme que, parmi les garçons, les amitiés sont plus nombreuses et qu'un effet d'homophilie en termes d'âge et de sexe s'observe, spécialement s'agissant des relations amicales (Bidart, 1997). Cependant, les résultats de l'étude présentée ici révèlent que les différences entre les hommes et les femmes, concernant la relation avec les pairs, ne sont pas globalement significatives.

Concernant le lieu de naissance, l'engagement scolaire des jeunes ne présente pas de différences statistiques globales entre les natifs et les immigrés, et ce dans aucune des dimensions analysées. De même, aucune différence statistiquement significative n'a été observée concernant le lieu de naissance de la mère, dans aucune des dimensions de l'engagement analysées.

La situation économique de la famille est un autre aspect analysé et on n'observe pas de différence statistiquement significative s'agissant des relations avec le personnel enseignant ou avec les camarades de classe (Voir Tableau 2 dans l'article).

Les opinions des jeunes quant aux attentes de la famille vis-à-vis de leur poursuite d'études diffèrent pour un seul item. Ainsi, il est intéressant de noter que les élèves rencontrant des difficultés économiques pensent qu'ils contrarieraient leurs parents s'ils abandonnaient leur formation, ce qui n'est pas le cas des élèves sans difficultés financières.

Cependant, les différences en fonction des difficultés économiques ne sont pas significatives s'agissant des attentes de la famille en termes de résultats scolaires de leurs enfants.

Finalement, des différences statistiquement significatives ont été observées pour les dimensions liées à la disponibilité et au soutien de la famille. Ainsi, les élèves ayant des difficultés économiques plus importantes bénéficieraient d'un moindre soutien familial. 
Tableau 2. Opinions sur l'engagement scolaire et la situation financière de la famille. Comparaison de moyennes

\begin{tabular}{|c|c|c|c|c|}
\hline & \multicolumn{4}{|c|}{ Situation financière de la famille } \\
\hline & Aucune difficulté & Peu de difficultés & $\begin{array}{l}\text { Nombreuses } \\
\text { difficultés }\end{array}$ & Total \\
\hline $\begin{array}{l}\text { Ma famille est disponible quand j'en } \\
\text { ai besoin }\end{array}$ & 2,65 & $2,51^{*}$ & $2,32^{*}$ & 2,53 \\
\hline $\begin{array}{l}\text { Quand quelque chose de positif } \\
\text { m'arrive au collège ou lycée, ma famille } \\
\text { veut être informée }\end{array}$ & 2,57 & $2,35^{*}$ & $2,33^{*}$ & 2,45 \\
\hline $\begin{array}{l}\text { Je peux compter sur mes parents si j'ai } \\
\text { des problèmes au collège ou lycée }\end{array}$ & 2,56 & 2,49 & 2,48 & 2,52 \\
\hline $\begin{array}{l}\text { Mes parents ou tuteurs sont informés } \\
\text { quand j'ai des dossiers à rendre ou des } \\
\text { examens }\end{array}$ & 1,66 & 1,61 & 1,43 & 1,59 \\
\hline $\begin{array}{l}\text { Mes parents font tout ce qu'ils peuvent } \\
\text { pour m'aider à obtenir de bonnes notes }\end{array}$ & 2,29 & $2,21^{*}$ & $1,94^{*}$ & 2,18 \\
\hline $\begin{array}{l}\text { Mes parents me demandent souvent } \\
\text { comment ça se passe au collège ou } \\
\text { au lycée }\end{array}$ & 2,50 & 2,51 & 2,37 & 2,46 \\
\hline $\begin{array}{l}\text { Disponibilité et soutien familial } \\
\text { (total) }\end{array}$ & 2,31 & $2,23^{*}$ & $2,11^{*}$ & 2,23 \\
\hline $\begin{array}{l}\text { Mes parents souhaitent que je } \\
\text { poursuive le plus longtemps possible } \\
\text { mes études }\end{array}$ & 2,61 & 2,61 & 2,53 & 2,59 \\
\hline $\begin{array}{l}\text { Pour mes parents, il est important que } \\
\text { je réussisse mon année }\end{array}$ & 2,82 & 2,78 & 2,68 & 2,77 \\
\hline $\begin{array}{l}\text { Mes parents seraient contrariés si } \\
\text { j'arrêtais les études }\end{array}$ & 2,37 & $2,65^{*}$ & $2,53^{*}$ & 2,48 \\
\hline $\begin{array}{l}\text { Pour mes parents, il est important que } \\
\text { je donne le meilleur de moi-même } \\
\text { dans les études }\end{array}$ & 2,74 & 2,63 & 2,63 & 2,68 \\
\hline $\begin{array}{l}\text { Attentes des parents en termes de } \\
\text { réussite scolaire (total) }\end{array}$ & $\begin{array}{c}2,64 \\
49 \% \\
(n=160)\end{array}$ & $\begin{array}{c}2,69 \\
23 \% \\
(n=76)\end{array}$ & $\begin{array}{c}2,60 \\
28 \% \\
(n=90)\end{array}$ & $\begin{array}{c}2,64 \\
100 \% \\
(n=326)\end{array}$ \\
\hline
\end{tabular}

Note : * les valeurs de la même ligne comportant un * et grisées sont significativement différentes à $p<, 05$ dans le test d'égalité bilatérale pour les moyennes en colonne. Les cellules sans indice ne sont pas incluses dans le test. Les tests supposent des variances égales.

Source : auteur.e.s. 
Pour le niveau d'études des parents, aucune différence statistiquement significative ne s'observe concernant l'engagement scolaire (Tableau 3 dans l'article). Cependant, des différences significatives ressortent pour certains éléments. En termes de relation avec les enseignants, les élèves dont les parents ont un niveau de diplôme moyen ou supérieur pensent davantage que les enseignants sont moins ouverts et honnêtes avec eux que les élèves dont les parents possèdent un niveau d'études de base. Il en va de même des attentes familiales. Ainsi, le taux d'élèves dont les parents ont un niveau d'études moyen ou supérieur et qui pensent qu'ils décevraient leurs parents s'ils abandonnaient leurs études est plus élevé que celui des élèves dont les parents ont un niveau d'éducation de base. De plus, les attentes des parents possédant un diplôme moyen ou supérieur, en termes de poursuite d'études, seraient plus élevées. Cependant, ces différences ne génèrent pas de disparités globales en termes d'attentes de réussite de la part des parents.

Tableau 3. Opinions sur l'engagement scolaire selon le niveau d'études des parents. Comparaison de moyennes

\begin{tabular}{|c|c|c|c|}
\hline & \multicolumn{3}{|c|}{ Niveau d'études des parents } \\
\hline & Faible & Moyen ou supérieur & Total \\
\hline $\begin{array}{l}\text { Mes professeurs sont disponibles quand j'en ai } \\
\text { besoin }\end{array}$ & 2,24 & 2,30 & 2,26 \\
\hline $\begin{array}{l}\text { Le personnel enseignant de mon collège ou lycée } \\
\text { écoute les élèves }\end{array}$ & 2,04 & 2,15 & 2,08 \\
\hline $\begin{array}{l}\text { Le personnel enseignant de mon établissement } \\
\text { s'intéresse à moi non seulement en tant qu'élève, } \\
\text { mais aussi en tant que personne }\end{array}$ & 1,88 & 1,84 & 1,86 \\
\hline $\begin{array}{l}\text { En général, mes professeurs sont ouverts et } \\
\text { honnêtes avec moi }\end{array}$ & 2,17 & $1,98^{*}$ & 2,10 \\
\hline $\begin{array}{l}\text { En général, le personnel enseignant de mon } \\
\text { collège ou lycée traite les élèves d'une manière } \\
\text { adéquate }\end{array}$ & 2,10 & 2,08 & 2,09 \\
\hline $\begin{array}{l}\text { J'aime parler aux professeurs de mon collège ou } \\
\text { lycée }\end{array}$ & 1,78 & 1,84 & 1,80 \\
\hline $\begin{array}{l}\text { Dans mon collège ou lycée, la plupart des } \\
\text { enseignants se préoccupent de leurs élèves }\end{array}$ & 1,96 & 2,08 & 2,00 \\
\hline Relations avec les enseignants (total) & 2,03 & 2,03 & 2,03 \\
\hline $\begin{array}{l}\text { Mes parents souhaitent que je continue le plus } \\
\text { longtemps possible mes études }\end{array}$ & 2,52 & $2,72^{*}$ & 2,60 \\
\hline $\begin{array}{l}\text { Pour mes parents, il est important que je réussisse } \\
\text { mon année }\end{array}$ & 2,78 & 2,79 & 2,79 \\
\hline $\begin{array}{l}\text { Mes parents seraient contrariés si j’arrêtais les } \\
\text { études }\end{array}$ & 2,40 & $2,63^{*}$ & 2,49 \\
\hline $\begin{array}{l}\text { Pour mes parents, il est important que je donne le } \\
\text { meilleur de moi-même dans les études }\end{array}$ & 2,70 & 2,69 & 2,70 \\
\hline $\begin{array}{l}\text { Attentes des parents en termes de réussite } \\
\text { scolaire (total) }\end{array}$ & $\begin{array}{c}2,62 \\
62 \% \\
(n=182) \\
\end{array}$ & $\begin{array}{c}2,71 \\
38 \% \\
(n=111)\end{array}$ & $\begin{array}{c}2,65 \\
100 \% \\
(n=293) \\
\end{array}$ \\
\hline
\end{tabular}

Note : * les valeurs de la même ligne comportant un * et grisées sont significativement différentes à $\mathrm{p}<, 05$ dans le test d'égalité bilatérale pour les moyennes en colonne. Les cellules sans indice ne sont pas incluses dans le test. Les tests supposent des variances égales. Source : auteur.e.s. 


\section{Discussion}

Lobjectif initial de l'article était, d'une part, de mieux comprendre l'intention de poursuivre les études en formation professionnelle de base d'un groupe d'élèves, à l'aune du soutien relationnel reçu de la part des parents, des amis et des enseignants ; d'autre part, de considérer l'influence des caractéristiques sociodémographiques comme un facteur explicatif du soutien relationnel à disposition de ces jeunes.

Les résultats soulignent une hétérogénéité de facteurs influençant l'engagement scolaire des jeunes, notamment la possibilité de trouver du soutien auprès des enseignants. Dans une moindre mesure, concernant l'impact des relations familiales, l'engagement dans les études dépend de certaines caractéristiques sociodémographiques (le niveau d'études de la mère et la situation financière de la famille).

Létude présente également des disparités s’agissant du soutien des amis, selon le genre. Ainsi, les garçons déclarent plus souvent avoir un réseau d'amis. À cet égard, nous faisons l'hypothèse que les jeunes garçons pourraient être davantage influencés que les filles par leurs pairs, que ce soit en termes de poursuite d'études ou d'intention d'abandonner. En ce sens, certains auteurs soulignent que les garçons ont de meilleures relations avec leurs camarades de classe que les filles, mais ces dernières ont tendance à faire un meilleur usage de leurs réseaux en termes de soutien scolaire (Elffers, op. cit.).

Le lieu de naissance et les origines familiales n'influencent pas les opinions sur l'engagement relationnel. Cette étude va ainsi à l'encontre des résultats d'autres auteurs (Rumberger \& Lim, 2008). Nous faisons l'hypothèse que nos résultats sont liés à la relative homogénéité de ces jeunes en situation défavorable et dont les caractéristiques sociodémographiques sont très spécifiques : parcours scolaires non linéaires et marqués par la rupture, jeunes issus de familles vulnérables et ayant suivi leurs études par défaut (Salvà-Mut, 2018 ; Cerdà-Navarro, 2019).

Par ailleurs, l'appartenance sociale conditionne le poids du soutien familial et des attentes des familles en termes de réussite scolaire. L'implication familiale reste tributaire du poids de la situation économique du foyer sur les items de disponibilité, de soutien familial et d'attentes de la famille en termes de réussite. Nous pourrions confirmer l'hypothèse de Rosenfeld \& al. (op. cit.) d'un meilleur engagement dans la formation de la part des jeunes bénéficiant du soutien familial. Selon cet auteur, s'y ajoute le soutien que les jeunes reçoivent de leurs enseignants et de leur groupe de pairs. Les jeunes issus de classes sociales défavorisées bénéficient d'un moindre soutien familial que leurs pairs issus de familles sans difficultés. En effet, l'importance d'obtenir de bonnes notes, l'intérêt de la famille pour ce qui peut se passer globalement au lycée ou collège, et la disponibilité de la famille en cas de besoin sont moindres dans les familles issues des classes sociales défavorisées. À l'inverse, l'abandon des études contrarie davantage les familles des jeunes issus de milieux économiques moyens ou élevés. 
Ainsi, le niveau d'études des parents influence les attentes des familles vis-à-vis de leurs enfants. Les enfants issus de familles où les parents possèdent un niveau d'études moyen ou supérieur déclarent davantage que les attentes familiales en termes de résultats scolaires et de poursuite des études sont importantes, et qu'ils contrarieraient plus leurs parents s'ils quittaient le système éducatif formel.

Quant à la recherche empirique existante dans ce domaine d'analyse, comme nous l'avons déjà mentionné, rares sont les études centrées sur la formation professionnelle de base et l'abandon survenant à ce niveau d'éducation. Dès lors, cet article fournit des informations sur un domaine relativement peu étudié. Ainsi, les résultats de ce travail semblent d'autant plus utiles que de manière originale, aucune des variables sociodémographiques ou familiales évaluées n'a d'influence significative.

Néanmoins, et de manière générale, on retrouve des différences parmi les quatre domaines relationnels étudiés en fonction des caractéristiques sociodémographiques (relations avec les enseignants, relations avec les pairs, disponibilité et soutien familial, attentes des parents en termes de réussite scolaire). Cela nous permet de conforter certains constats issus de la littérature. Ainsi, l'étude menée nous permet globalement de confirmer que les élèves qui bénéficient d'un fort soutien relationnel (famille, amis et enseignants) consacrent plus de temps aux études, évitent les comportements susceptibles de faire obstacle à leur poursuite d'études, se montrent plus autonomes et engagés dans leur formation (Rosenfeld \& al., op. cit.).

Le fait qu'une grande partie des items ne présentent pas de différences significatives s'explique par les caractéristiques générales de la population qui suit une formation professionnelle de base. Comme indiqué dans la partie de l'article consacrée à la méthodologie, la population est relativement homogène et des caractéristiques liées au décrochage sont surreprésentées. Il s'agit d'une population jeune, défavorisée, issue de familles possédant un faible niveau socio-économique, avec une forte proportion de mères nées dans des pays du sud non européens. Ce sont des élèves à la biographie scolaire marquée par de faibles résultats scolaires et de forts taux de redoublement et de ruptures et qui ont opéré ces choix de formation par défaut (Salvà-Mut. \& al., 2018). Pour autant, les résultats présentés ici révèlent que les jeunes n'ayant pas envisagé d'abandonner leurs études entretiennent une meilleure relation avec les enseignants. Le soutien des enseignants est positivement associé à l'implication comportementale à différents stades de l'éducation ${ }^{17}$ (Elffers \& al., 2012).

Afin de poursuivre les analyses, il serait intéressant de mener une comparaison longitudinale des différentes vagues de l'enquête (2015, 2016 et 2017), travail qui est en cours.

17. Voir la partie 1.2 pour plus de précisions sur l'influence des enseignants en termes de réduction du décrochage scolaire des étudiants. 
On pourrait aussi comparer les différents niveaux d'éducation et démontrer la relation entre les raisons de l'abandon et l'impact des motifs du choix de la formation, afin de mieux comprendre comment renforcer l'engagement des élèves. Ainsi, il serait utile de croiser les réponses au questionnaire et les abandons d'études constatés, ainsi que le devenir sur le marché de l'emploi (par exemple pendant la saison touristique aux Baléares avec les nombreux emplois peu qualifiés). Dès lors, il serait possible de mesurer le rôle démobilisateur des emplois saisonniers (effet de forte attractivité vers le monde du travail).

En parallèle, il conviendrait d'approfondir qualitativement les caractéristiques individuelles, afin de saisir de manière plus qualitative et compréhensive les effets de la biographie dans la décision de commencer et de poursuivre des études en formation professionnelle.

Notre volonté est donc de poursuivre cette étude pour vérifier si les variables significatives sont les mêmes et ont un poids similaire lorsque l'abandon des études est définitif.

Dès lors, en l'absence d'impact direct de la plupart des caractéristiques sociodémographiques sur les parcours des élèves déclarant avoir songé à abandonner leurs études, une des pistes pour atteindre l'objectif initial de cette recherche serait d'explorer davantage encore certains facteurs liés à l'engagement scolaire (cf. Reschly \& Christenson, op. cit), qui ont montré une influence sur le décrochage, tels les aspects relationnels avec les enseignants, dont l'impact est central sur les processus de persévérance scolaire et de poursuite d'études.

\section{Bibliographie}

Appleton J. J., Christenson S. L. \& Furlong M. J. (2008), "Student engagement with school: Critical conceptual and methodological issues of the construct", Psychology in the Schools, 45(5), pp. 369-386.

Appleton J.J. (2012). "Systems Consultation: Developing the Assessment-to-Intervention Link with the Student Engagement Instrument”, in Christentson S.L. ; Reschly A. \& Wylie C. (ed.), Handbook of Research on Student Engagement. NY: Springer, pp. 725-742.

Archambault I., Janosz M., Morizot J. \& Pagani, L. (2009), “Adolescent behavioral, affective, and cognitive engagement in school: Relationship to drop-out", Journal of School Health, 79(9), pp. 408-415. https://doi.org/10.1111/j.1746-1561.2009.00428.x

Barry M. \& Reschly A. L. (2012), "Longitudinal predictors of high school completion", School Psychology Quarterly, 27(2), p. 74.

Beaton A.M. \& Beaton A. (2019), "Building an engaging community: Practices and interventions that support students in schools", in Fredricks J.A. ; Reschly A.L. \& Christenson S.L. (ed.), Handbook of student engagement interventions. Elsevier, pp. 345358. https://doi.org/10.1016/B978-0-12-813413-9.00023-1 
Bidart C. (1997), L'amitié, un lien social, Paris, La Découverte.

Brewster A. B. \& Bowen G. L. (2004), “Teacher support and the school engagement of Latino middle and high school students at risk of school failure", Child and Adolescent Social Work Journal, 21(1), pp. 47-67.

Cerdà-Navarro A., Sureda-Negre J. \& Comas-Forgas R. (2017), "Recommendations for confronting vocational education dropout: a literature review", Empirical Research in Vocational Education \& Training, pp. 9-17. https://doi.org/10.1186/ s40461-017-0061-4

Cerdà-Navarro A. (2019), Abandono educativo temprano en la formación profesional de grado medio. Thèse de doctorat en sciences de l'éducation non publiée. Universitat de les Illes Balears, Palma (Balears), España.

Cerdà-Navarro A., Salva-Mut F. \& Comas Forgas R. (2019), "A typology of students in intermediate vocational education and training programmes based on student engagement factors, sociodemographic characteristics and intentions of dropping out”, European Journal of Education, 54(4), pp. 635-650. https://doi.org/10.1111/ ejed. 12361

Cerdà-Navarro A., Salvà-Mut F. \& Comas-Forgas R., (2020), "Intención de abandono y abandono durante el primer curso de Formación Profesional de Grado Medio: una análisis tomando como referencia el concepto de implicación del estudiante (student engagement)", Estudios de Educación, 39 (In press).

Christenson S. L. \& Thurlow M. L. (2004), "School dropouts: Prevention considerations, interventions, and challenges", Current Directions in Psychological Science, 13(1), pp. 36-39.

Cohen-Scali V. (2003), “The influence of Family, Social and Work Socialization on the Construction of the Professional Identity of Young Adults ", Journal of Career Development, 29, pp. 237-249.

Cordero J.M., Manchón C. \& Simancas R., (2014), "La repetición de curso y sus factores condicionantes en España", Revista de Educación, 365, pp. 12-37.

Elffers L., Oort F. J. \& Karsten S. (2012), "Making the connection: The role of social and academic school experiences in students' emotional engagement with school in post-secondary vocational education", Learning and Individual Differences, 22(2), pp. 242-250.

Ferrand A. (2006), "Redes heterogéneas de discusión y pluralismo cognitive", Redes. Revista Hispana para el análisis de redes sociales (010), $V$.

Fredricks J. A., Blumenfeld P. C. \& Paris A. H. (2004), "School engagement: potential of the concept, state of the evidence", Review of Educational Research, 74(1), pp. 59-109. 
Furrer C. \& Skinner E. (2003), "Sense of relatedness as a factor in children's academic engagement and performance", Journal of Educational Psychology, 95(1), pp. 148-162.

Janosz M., Archambault I., Lacroix M. \& Lévesque J. (2007), Trousse d'évaluation des décrocheurs potentiels (TEDP) : Manuel d'utilisation. Montréal : Groupe de recherche sur les environnements scolaires. Université de Montréal.

Jimerson S. R., Ferguson P., Whipple A. D., Anderson G. E \& Dalton M. J. (2002), "Exploring the association between grade retention and dropout: A longitudinal study examining socio-emotional, behavioral, and achievement characteristics of retained students", The California School Psychologist, 7, pp. 51-62.

Knox H., Savag M. \& Harvey P. (2006), "Social networks and the study of relations: networks as method, metaphor and form", Economy and Society, 35(1), pp. 113-140.

Lannegrand L., Cosnefroy O. \& Lecigne A. (2012), "Prediction of various degrees of vocational secondary school absenteeism: Importance of the organization of the educational system", School Psychology International, 33:94, pp. 293-307.

Mackay K. R. (2005), Prediction of Dropout in a Residential Vocational Education Institution, Thèse de doctorat en philosophie et ssychologie des organizations, Alliant International University, California School of Business and Organizational Studies and the California School of Professional Psychology, San Diego.

Marti J. \& Lozares C. (2008), "Redes organizativas locales y capital social: enfoques complementarios desde el análisis de redes sociales", Portularia: Revista de Trabajo Social, (8), pp. 23-3.

Marhuenda-Fluixà F., Salvà-Mut F., Navas Saurín A. \& Abiétar López M. (2015), "Twenty Years of Basic Vocational Education Provision in Spain: Changes and Trends", International Journal for Research in Vocational Education and Training (IRJVET), 2(2), pp. 137-151.

MECD (Ministry of Education, Culture and Sport) (2010), Datos y Cifras.

MEFP (2019), Sistema estatal de indicadores de la educación 2019, Madrid: Ministerio de Educación y Formación Profesional.

Ministère de l'Éducation nationale et de la Jeunesse. (2020), Les définitions des termes et indicateurs statistiques de l'éducation nationale. https://www.education.gouv.fr/ les-definitions-des-termes-et-indicateurs-statistiques-de-l-education-nationale-5123?

Nouwen W. \& Clycq N. (2019), "The role of social support in fostering school engagement in urban schools characterised by high risk of early leaving from education and training", Social Psychology of Education. https://doi.org/10.1007/s11218-019-09521-6 
Passy F. (2002), "Social Networks Matter. But How?, in Diani M. \&. McAdam D. (Eds.), Social Movement Analysis: The Network Perspective. Oxford: Oxford University Press.

Reschly A. \& Christenson S. L. (2012), "Jingle, jangle, and conceptual haziness: Evolution and future directions of the engagement construct", in Christentson S. L., Reschly A \& Wylie C. (Eds.), Handbook of research on student engagemen, pp. 3-20. New York: Springer. https:// doi.org/10.1007/978-1-4614-2018-7_1

Roorda D. L., Koome H. M., Spilt J. L. \& Oort F. J. (2011), "The influence of affective teacher-student relationships on students' school engagement and achievement: A meta-analytic approach”, Review of Educational Research, 81(4), pp. 493-529.

Rosenfeld L. B., Richman J. M. \& Bowen G. L. (2000), "Social support networks and school outcomes: The centrality of the teacher", Child and Adolescent Social Work Journal, 17(3), pp. 205-226.

Rumberger R. W. \& Lim S. A. (2008), "Why students drop out of school? A review of 5 years of research", California Dropout Research Project, 15, pp. 1-3.

Salinas J. \& Santín D., ( 2012), "Selección escolar y efectos de la inmigración sobre los resultados académicos españoles en PISA 2006”, Revista de Educación, 358, pp. 382-405.

Salvà-Mut F., Thomàs-Vanrell C., Calvo-Sastre A. \& Pinya-Medina C. (2018), Inclusion and exclusion in Basic VET programmes in Spain, in VETNET Network ECER 2018 ECER, 4-7 septembre, Bolzano.

Salvà-Mut F., Cerdà-Navarro A. \& Sureda-Negre J. (2019), “The Promotion ofEducational Success in Intermediate Level VET:The Case of the Balearic Islands", in MarhuendaFluixá F. (eds), The School-Based Vocational Education and Training System in Spain. Technical and Vocational Education and Training: Issues, Concerns and Prospects, vol. 32, pp. 167-186. Springer, Singapore. https://doi.org/10.1007/978-981-13-8475-2_9

Stearns E., Moller S., Blau J. \& Potochnick S. (2007), « Staying back and dropping out: The relationship between grade retention and school dropout ", Sociology of Education, 80(3), pp. 210-240.

Vonthron A-M., Lagabrielle C. \& Pouchard D. (2007), " Le maintien en formation professionnelle qualifiante : effets de déterminants motivationnels, cognitifs et sociaux", L'orientation scolaire et professionnelle, 36/3, pp. 401-420.

Wang M. T. \& Eccles J. S. (2012), “Social support matters: Longitudinal effects of social support on three dimensions of school engagement from middle to high school”, Child Development, 83(3), pp. 877-895. 